\title{
ON THE CURVATURE AND TORSION EFFECTS IN ONE DIMENSIONAL WAVEGUIDES
}

\author{
Guy Bouchitté ${ }^{1}$, M. Luísa Mascarenhas ${ }^{2}$ and Luís Trabucho ${ }^{3}$
}

\begin{abstract}
We consider the Laplace operator in a thin tube of $\mathbb{R}^{3}$ with a Dirichlet condition on its boundary. We study asymptotically the spectrum of such an operator as the thickness of the tube's cross section goes to zero. In particular we analyse how the energy levels depend simultaneously on the curvature of the tube's central axis and on the rotation of the cross section with respect to the Frenet frame. The main argument is a $\Gamma$-convergence theorem for a suitable sequence of quadratic energies.
\end{abstract}

Mathematics Subject Classification. 49R50, 35P20, 78A50, 81Q15.

Received February 23, 2006. Revised July 7, 2006.

Published online September 5, 2007.

\section{INTRODUCTION}

We are interested in the 3D-1D reduction analysis for the following elementary spectral problem:

$$
-\Delta u_{\varepsilon}=\lambda^{\varepsilon} u_{\varepsilon}, \quad u_{\varepsilon} \in H_{0}^{1}\left(\Omega_{\varepsilon}\right),
$$

where $\Omega_{\varepsilon} \subset \mathbb{R}^{3}$ is a thin and long domain generated by a cross section $\omega_{\varepsilon}=\varepsilon \omega\left(\omega \subset \mathbb{R}^{2}\right)$ which rotates along a curve $r(s) \in \mathbb{R}^{3}$ parametrized by $s$, the usual arc length variable. Here $\varepsilon$ is a small parameter and the rotation angle $\alpha(s)$ of the section with respect to the Frénet frame is given. We are going to show the following behavior of the spectrum $\left\{\lambda_{i}^{\varepsilon} ; i \in \mathbb{N}\right\}$ as $\varepsilon \rightarrow 0$ :

$$
\lambda_{i}^{\varepsilon}=\frac{\lambda_{0}}{\varepsilon^{2}}+\mu_{i}^{\varepsilon}, \quad \mu_{i}^{\varepsilon} \rightarrow \mu_{i}
$$

where $\lambda_{0}$ is the first eigenvalue of the Laplace operator on $\omega$ and the $\mu_{i}$ 's are the eigenvalues of a one dimensional problem of the kind

$$
-w^{\prime \prime}+q(s) w=\mu w, \quad w \in H_{0}^{1}(0, L) .
$$

Here $q(s)$ is an effective potential which we are able to characterize in terms of the parameters $k(s), \tau(s)$ (curvature and torsion), of the shape of $\omega$ and of the rotation angle $\alpha(s)$.

\footnotetext{
Keywords and phrases. Dimension reduction, $\Gamma$-convergence, curvature and torsion, waveguides.

1 Département de Mathématiques, Université du Sud-Toulon-Var, BP 132, 83957 La Garde Cedex, France; bouchitte@univ-tln.fr

2 Departamento de Matemática da F.C.T.-U.N.L. e C.M.A.-U.N.L., Quinta da Torre, 2829-516 Caparica, Portugal; mlfm@fct.unl.pt

3 Departamento de Matemática da F.C.-U.L. e C.M.A.F.-U.L., Av. Prof. Gama Pinto 2, 1649-003 Lisboa, Portugal; trabucho@ptmat.fc.ul.pt
} 
A possible physical motivation for this problem is the understanding of the behavior of the probability density associated with the wave function of a particle confined in a thin waveguide. The interpretation of the convergence result above is that, from the particle's point of view, everything happens as if it will propagate in a one dimensional medium governed by the non zero potential $q(s)$. Several results have been published in this direction, for instance in $[2,5]$, in the case of tube of infinite length with circular cross section, showing the shift of the spectrum on the left due to the curvature and the possible occurence of localized modes. Here we emphasize on the effects of the torsion and of the shape of cross section which, in the opposite direction, tend to shift the spectrum on the right. Moreover we present a new rigourous variational approach through $\Gamma$-convergence which is very flexible and can be adapted to other kind of spectral problems with possibly stiff parameters.

Let us emphasize that the geometrical effects described here are very specific to the Dirichlet boundary condition imposed on the lateral part of the tube. Such effects would disappear if the Dirichlet condition would be replaced by a Neumann condition. We refer to [8] (see also the survey [7]) for related questions where networks of tubes with junctions are considered. Let us finally mention a pioneering work by M. Vanninathan [9] where a behavior of the kind (1.2) was established for the eigenvalues of the Dirichlet problem on a periodically perforated domain as the period $\varepsilon$ tends to zero.

In Section 2 we describe the geometric properties of the domain. In Section 3, we present the rescaled spectral problem on a varying Hilbert space and show how the asymptotic behavior of the entire spectrum can be recovered by proving the $\Gamma$-convergence of a suitable family of quadratic energies. In Section 4 , we preliminary study a perturbed problem for the first eigenvalue in the cross section and then establish the main convergence result. Eventually, some elementary examples of limit models are discussed in Section 5.

\section{Geometry of the domain}

Let $r: s \in[0, L] \rightarrow r(s) \in \mathbb{R}^{3}$ be a simple $C^{2}$ curve in $\mathbb{R}^{3}$ parametrized by the arc length parameter $s$. Denoting by $T$ its tangent vector and assuming that $T^{\prime}(s) \neq 0$ for every $s \in[0, L]$, we may define the usual Frenet system $(T, N, B)$ through the following expressions:

$$
T=\frac{\mathrm{d} r}{\mathrm{~d} s}=r^{\prime} \quad\left(\left\|r^{\prime}\right\|_{\mathbb{R}^{3}}=1\right) ; \quad N=T^{\prime} /\left\|T^{\prime}\right\|_{\mathbb{R}^{3}} ; \quad B=T \times N .
$$

Denote by $k: s \in[0, L] \rightarrow k(s) \in \mathbb{R}$ and by $\tau: s \in[0, L] \rightarrow \tau(s) \in \mathbb{R}$, the curvature and torsion functions associated with the curve. They are functions in $L^{\infty}(0, L)$ and they satisfy the Frenet formulas:

$$
T^{\prime}=k N ; \quad N^{\prime}=-k T+\tau B ; \quad B^{\prime}=-\tau N .
$$

Let now $\omega \subset \mathbb{R}^{2}$ be an open bounded, simply connected subset of $\mathbb{R}^{2}$ and consider the following subset of $\mathbb{R}^{3}$, directly associated with the Frenet system defined above:

$$
\Omega^{F}=\left\{x \in \mathbb{R}^{3}: x=r(s)+y_{1} N(s)+y_{2} B(s), s \in[0, L], y=\left(y_{1}, y_{2}\right) \in \omega\right\} .
$$

However this choice is too restrictive as we may like that the possibly non circular cross section $\omega$ of our waveguide rotates with respect to curve $r$ in a different way as Frenet frame does. In particular another reference system, denominated Tang's reference system, could be considered as well, in which the corresponding domain:

$$
\Omega^{T}=\left\{x \in \mathbb{R}^{3}: x=r(s)+y_{1} X(s)+y_{2} Y(s), s \in[0, L], y=\left(y_{1}, y_{2}\right) \in \omega\right\},
$$

is such that its cross section is rotation free with respect to the tangent vector $T$. The orthonormal basis vectors of Tang's reference system are given by:

$$
X^{\prime}=\lambda T ; \quad Y^{\prime}=\mu T ; \quad T^{\prime}=-\lambda X-\mu Y ;
$$




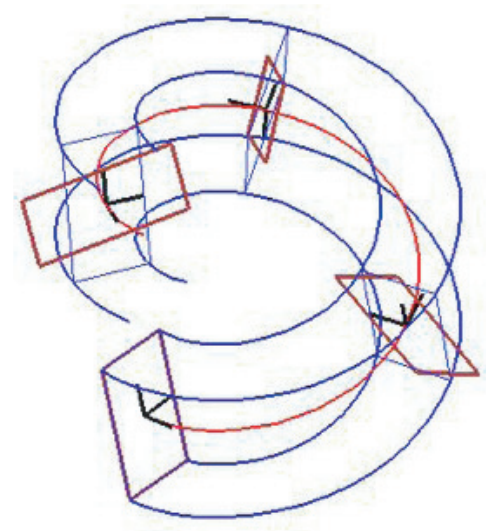

FiguRE 1. Reference domains associated with Frenet's and Tang's systems.

where $\lambda$ and $\mu$ are functions of the arc length parameter $s$. In Figure 1 we show an illustration of the domains $\Omega^{F}$ and $\Omega^{T}$ (four cross sections only).

For each $s \in[0, L]$ Tang's reference system is such that $(X, Y)$ can be seen as a two dimensional basis, in $\omega$, rotated from $(N, B)$, around $T$, of an angle $\alpha=\alpha(s)$. In fact if

$$
X=\cos \alpha N+\sin \alpha B, \quad Y=-\sin \alpha N+\cos \alpha B
$$

using Frenet's formulas (2.1), one obtains:

$$
\begin{aligned}
& X^{\prime}=-\left(\tau+\alpha^{\prime}\right) \sin \alpha N+\left(\tau+\alpha^{\prime}\right) \cos \alpha B-k \cos \alpha T, \\
& Y^{\prime}=-\left(\tau+\alpha^{\prime}\right) \cos \alpha N-\left(\tau+\alpha^{\prime}\right) \sin \alpha B+k \sin \alpha T,
\end{aligned}
$$

which yields (2.2) if, for each $s \in[0, L], \alpha(s)$ satisfies the so called Tang's relations:

$$
\alpha^{\prime}=-\tau, \quad \lambda=-k \cos \alpha, \quad \mu=k \sin \alpha .
$$

We notice that, in contrast with Frenet's system, the condition $T^{\prime}(s) \neq 0$ is not required in order to construct the Tang basis $(T, X, Y)$ (which is uniquely determined as a solution of $(2.2)$ ).

We are then faced with many possible choices for the reference set and, in order to model a general twisted tube, we will consider the generic domain $\Omega^{\alpha}$ defined through:

$$
\Omega^{\alpha}=\left\{x \in \mathbb{R}^{3}: x=r(s)+y_{1} N_{\alpha}(s)+y_{2} B_{\alpha}(s), s \in[0, L], y=\left(y_{1}, y_{2}\right) \in \omega\right\}
$$

whose cross section presents an arbitrary rotation of an angle $\alpha$ with respect to Frenet's domain:

$$
\begin{aligned}
& N_{\alpha}(s):=\cos \alpha(s) N(s)+\sin \alpha(s) B(s), \\
& B_{\alpha}(s):=-\sin \alpha(s) N(s)+\cos \alpha(s) B(s) .
\end{aligned}
$$

As is clear from the above notation, if for every $s \in[0, L], \alpha=0$ then $\Omega^{\alpha} \equiv \Omega^{F}$ and if $\alpha$ is such that $\alpha^{\prime}=-\tau$, then $\Omega^{\alpha}=\Omega^{T}$.

We are interested in the (eigenvalue) problem given by (1.1) in a thin domain such that the diameter of the cross section $\omega$ is much smaller than its length $L$. Specifically, we consider a real parameter $\varepsilon>0$ and a cross section, obtained from the reference one, by an homothety of ratio $\varepsilon$. Our thin waveguide will be then 


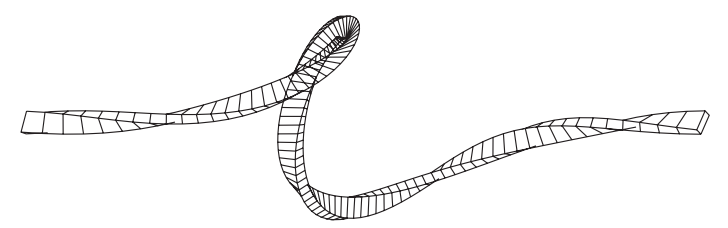

FiguRE 2. A generic domain $\Omega_{\varepsilon}^{\alpha}$.

determined as follows (Fig. 2):

$$
\Omega_{\varepsilon}^{\alpha}:=\left\{x \in \mathbb{R}^{3}: x=r(s)+\varepsilon y_{1} N_{\alpha}+\varepsilon y_{2} B_{\alpha}, s \in[0, L], y=\left(y_{1}, y_{2}\right) \in \omega\right\}
$$

\section{Asymptotic SPECTRAL PROBlem AND $\gamma$-CONVERGENCE APPROACH}

We consider, for fixed $\varepsilon>0$, the thin domain $\Omega_{\varepsilon}^{\alpha}$ defined in Section 2 and the following eigenvalue problem

$$
\left\{\begin{array}{l}
-\Delta u_{\varepsilon}=\lambda^{\varepsilon} u_{\varepsilon} \\
u_{\varepsilon} \in H_{0}^{1}\left(\Omega_{\varepsilon}^{\alpha}\right) .
\end{array}\right.
$$

As $\Omega_{\varepsilon}^{\alpha}$ is bounded, the spectrum $\sigma^{\varepsilon}$ of this problem is discrete and can be written as $\sigma^{\varepsilon}:=\left\{\lambda_{i}^{\varepsilon}: i \in \mathbb{N}\right\}$, where $0<\lambda_{0}^{\varepsilon} \leq \lambda_{1}^{\varepsilon} \leq \cdots \leq \lambda_{i}^{\varepsilon} \leq \lambda_{i+1}^{\varepsilon} \cdots$ are positive reals, arranged increasingly. As the cross section becomes thiner and thiner, it is clear that all these eigenvalues go to infinity as $\varepsilon \rightarrow 0$. More precisely, let $\lambda_{0}$ be the fundamental eigenvalue for the Laplace operator in the cross section $\omega$ and let $u_{0}$ be the associated normalized eigenvector, that is

$$
-\Delta u_{0}=\lambda_{0} u_{0}, \quad u_{0} \in H_{0}^{1}(\omega), \quad u_{0}>0, \quad \int_{\omega} u_{0}^{2}=1
$$

We are expecting the following asymptotic behavior:

$$
\lambda_{i}^{\varepsilon}=\frac{\lambda_{0}}{\varepsilon^{2}}+\mu_{i}+\rho(\varepsilon), \quad \lim _{\varepsilon \rightarrow 0} \rho(\varepsilon)=0,
$$

where $\mu_{i}(i \in \mathbb{N})$ are suitable real numbers. Our goal is to establish (3.3) and to identify the set $\left\{\mu_{i}\right\}$ as the eigenvalues of a one dimensional spectral problem in $H_{0}^{1}(0, L)$ in which the geometric parameters $k(s), \tau(s), \alpha(s)$ are involved.

\subsection{Change of variables}

As usual in the dimension reduction analysis, we first proceed to a rescaling and a change of variables in order to reduce the initial problem to a variational min-max formulation on a fixed domain. Having in mind the asymptotic behavior of the shifted spectrum $\sigma^{\varepsilon}-\frac{\lambda_{0}}{\varepsilon^{2}}$, the initial quadratic energy defined in $H_{0}^{1}\left(\Omega_{\varepsilon}^{\alpha}\right)$ reads as:

$$
F_{\varepsilon}(w):=\int_{\Omega_{\varepsilon}^{\alpha}}\left(|\nabla w|^{2}-\frac{\lambda_{0}}{\varepsilon^{2}}|w|^{2}\right) \mathrm{d} x .
$$

Recalling (2.3), consider the following transformation, for each $\varepsilon>0$,

$$
\begin{aligned}
& \psi_{\varepsilon}:[0, L] \times \omega \longrightarrow \Omega_{\varepsilon}^{\alpha} \\
& \left(s,\left(y_{1}, y_{2}\right)\right) \mapsto x=r(s)+\varepsilon y_{1} N_{\alpha}+\varepsilon y_{2} B_{\alpha}
\end{aligned}
$$

and define, for each $w \in H_{0}^{1}\left(\Omega_{\varepsilon}^{\alpha}\right), v\left(s,\left(y_{1}, y_{2}\right)\right):=w\left(\psi_{\varepsilon}\left(s,\left(y_{1}, y_{2}\right)\right)\right)$. 
We obtain that, in the Frénet frame:

$$
\nabla \psi_{\varepsilon}=\left(\begin{array}{ccc}
\beta_{\varepsilon} & 0 & 0 \\
-\varepsilon\left(\tau+\alpha^{\prime}\right)\left(z_{\alpha}^{\perp} \cdot y\right) & \varepsilon \cos \alpha & -\varepsilon \sin \alpha \\
\varepsilon\left(\tau+\alpha^{\prime}\right)\left(z_{\alpha} \cdot y\right) & \varepsilon \sin \alpha & \varepsilon \cos \alpha
\end{array}\right), \quad \operatorname{det} \nabla \psi_{\varepsilon}=\varepsilon^{2} \beta_{\varepsilon}
$$

where $z_{\alpha}, z_{\alpha}^{\perp}$ and $\beta_{\varepsilon}$ are given by

$$
\beta_{\varepsilon}(s, y):=1-\varepsilon k(s)\left(z_{\alpha} \cdot y\right), \quad z_{\alpha}:=(\cos \alpha,-\sin \alpha), \quad z_{\alpha}^{\perp}:=(\sin \alpha, \cos \alpha),
$$

and where, as previously, $\alpha^{\prime}$ represents the derivative of $\alpha$ with respect to $s \in[0, L]$.

Then

$$
\nabla \psi_{\varepsilon}^{-1}=\left(\begin{array}{ccc}
\frac{1}{\beta_{\varepsilon}} & 0 & 0 \\
\frac{\left(\tau+\alpha^{\prime}\right) y_{2}}{\beta_{\varepsilon}} & \frac{\cos \alpha}{\varepsilon} & \frac{\sin \alpha}{\varepsilon} \\
\frac{-\left(\tau+\alpha^{\prime}\right) y_{1}}{\beta_{\varepsilon}} & \frac{-\sin \alpha}{\varepsilon} & \frac{\cos \alpha}{\varepsilon}
\end{array}\right) .
$$

Denote $v(s, y)=w\left(\psi_{\varepsilon}(s, y)\right)$, for $w \in H_{0}^{1}\left(\Omega_{\varepsilon}^{\alpha}\right), s \in[0, L]$ and $y \in \omega$. We scale the functional $F_{\varepsilon}$ introduced in (3.4) by dividing it by $\varepsilon^{2}$. We are led to the quadratic energy $G_{\varepsilon}$ defined by:

$$
G_{\varepsilon}(v):=\frac{1}{\varepsilon^{2}} F_{\varepsilon}(v)=\int_{0}^{L} \int_{\omega}\left\{\frac{1}{\beta_{\varepsilon}}\left|v^{\prime}+\nabla_{y} v \cdot R y\left(\tau+\alpha^{\prime}\right)\right|^{2}+\frac{\beta_{\varepsilon}}{\varepsilon^{2}}\left(\left|\nabla_{y} v\right|^{2}-\lambda_{0}|v|^{2}\right)\right\} \mathrm{d} y \mathrm{~d} s
$$

where $v^{\prime}$ stands for the derivative of $v$ in order to $s, \nabla_{y} v$ for the derivative of $v$ in order to $y$ and $R$ for the rotation matrix $\left(\begin{array}{cc}0 & 1 \\ -1 & 0\end{array}\right)$.

\subsection{The rescaled spectral problem}

Denote $Q_{L}=\omega \times(0, L)$ and let $H_{\varepsilon}$ be the Hilbert space $L^{2}\left(Q_{L}, \beta_{\varepsilon}\right)$ equipped with the weighted scalar product

$$
(u \mid v)_{\varepsilon}:=\int_{Q_{L}} u(x) v(x) \beta_{\varepsilon}(x) \mathrm{d} x .
$$

By (3.5) and since the curvature $k(s)$ is assumed to be bounded, $\beta_{\varepsilon}(s, y)$ converges uniformly to 1 as $\varepsilon \rightarrow 0$. Therefore all spaces $H_{\varepsilon}$ are topologically equivalent and the strong convergence in $H_{\varepsilon}$ is equivalent to the convergence in the fixed space $H:=L^{2}\left(Q_{L}\right)$.

Now we define $A_{\varepsilon}$ to be the unique closed self adjoint operator from $H_{\varepsilon}$ to $H_{\varepsilon}$ with dense domain $D\left(A_{\varepsilon}\right)=$ $H^{2}\left(Q_{L}\right) \cap H_{0}^{1}\left(Q_{L}\right)$ such that

$$
\left(A_{\varepsilon} v \mid v\right)_{\varepsilon}=G_{\varepsilon}(v), \quad \forall v \in D\left(A_{\varepsilon}\right)
$$

In view of (3.3) and (3.6), it turns out that $u_{\varepsilon} \in H_{0}^{1}\left(\Omega_{\varepsilon}^{\alpha}\right)$ solves the spectral equation $-\Delta u_{\varepsilon}=\lambda_{i}^{\varepsilon} u_{\varepsilon}$ if and only if the function $v_{\varepsilon}(s, y)=u_{\varepsilon} \circ \psi_{\varepsilon}(s, y)$ satisfies

$$
A_{\varepsilon} v_{\varepsilon}=\mu_{\varepsilon}^{i} v_{\varepsilon}, \quad v_{\varepsilon} \in D\left(A_{\varepsilon}\right)
$$

It turns out that the sequence $\left\{A_{\varepsilon}\right\}$ is not uniformly bounded and also that the related spectral problem involves a varying scalar product. Therefore it is not possible to use directly an approach through $H$-convergence and apply the general results to be found in [1]. As our operator becomes stiff as $\varepsilon \rightarrow 0$, we adopt a slightly different point of view, reducing the original asymptotic spectral problem to the study of the $\Gamma$-convergence of the sequence of functionals $\left\{G_{\varepsilon}\right\}$ on the fixed space $H=L^{2}\left(Q_{L}\right)$. 


\subsection{Link with the $\Gamma$-convergence theory}

First we extend the quadratic functional $G_{\varepsilon}$ given in (3.6) by setting $G_{\varepsilon}(v)=+\infty$ if $v \in L^{2}\left(Q_{L}\right) \backslash H_{0}^{1}\left(Q_{L}\right)$. We say that the sequence $\left\{G_{\varepsilon}\right\} \Gamma$-converges to $G$ in $H=L^{2}\left(Q_{L}\right)$ if the two following conditions hold:

(i) for any $v$ and $\left\{v_{\varepsilon}\right\}$ such that $v_{\varepsilon} \rightarrow v$ in $H, \liminf _{\varepsilon \rightarrow 0} G_{\varepsilon}\left(v_{\varepsilon}\right) \geq G(v)$;

(ii) for every $v$, there exists a sequence $\left\{\tilde{v}_{\varepsilon}\right\}$ such that $\tilde{v}_{\varepsilon} \rightarrow v$ in $H$ and $\lim _{\varepsilon \rightarrow 0} G_{\varepsilon}\left(\tilde{v}_{\varepsilon}\right)=G(v)$.

It turns out that such a $\Gamma$-limit $G$ always exists, possibly after extracting a subsequence. Also the $\Gamma$-convergence of $\left\{G_{\varepsilon}\right\}$ is unchanged if we substitute $G_{\varepsilon}$ by its lower semicontinuous envelope (with respect to the strong topology in $H$ ) and the $\Gamma$-limit $G$ enjoys the lower semicontinuity property as well. For further features on $\Gamma$-convergence theory, we refer to the monograph by Dal Maso [4], in which particular issues concerning the case of quadratic functionals and related linear operators are detailed (see Sect. 12 in this book). We have the following abstract result:

Theorem 3.1. Let $A_{\varepsilon}: H_{\varepsilon} \rightarrow H_{\varepsilon}$ be a sequence of densily defined self-adjoint operators where $H_{\varepsilon}$ coincides algebraically with a fixed Hilbert space $H$ endowed with a scalar product $(\cdot \cdot \cdot)_{\varepsilon}$ such that

$$
a_{\varepsilon}\|u\|^{2} \leq(u \mid u)_{\varepsilon} \leq b_{\varepsilon}\|u\|^{2},
$$

being $a_{\varepsilon}, b_{\varepsilon}$ suitable constants such that $a_{\varepsilon}, b_{\varepsilon} \rightarrow 1$.

Let $G_{\varepsilon}: H \rightarrow(-\infty,+\infty]$ be defined by $G_{\varepsilon}(v):=\left(A_{\varepsilon} v \mid v\right)_{\varepsilon}$ if $v \in D\left(A_{\varepsilon}\right)$, and $G_{\varepsilon}(v):=+\infty$ otherwise, and assume that the three following conditions hold:

(i) (Lower bound) $G_{\varepsilon}(v) \geq-c_{0}\|v\|^{2}$ for a suitable constant $c_{0} \geq 0$.

(ii) (Compactness) If $\sup _{\varepsilon} G_{\varepsilon}\left(v_{\varepsilon}\right)<+\infty$ and $\sup _{\varepsilon}\left\|v_{\varepsilon}\right\|+<+\infty$, then $\left\{v_{\varepsilon}\right\}$ is strongly relatively compact in $H$.

(iii) $G_{\varepsilon}$ does $\Gamma$-converge to $G$.

Then the limit functional $G$ determines a unique closed linear operator $A_{0}: H \rightarrow H$ with compact resolvent (whose domain $D\left(A_{0}\right)$ is a priori non dense in $H$ ) such that $G(v)=\left(A_{0} v \mid v\right)$ for all $v \in D\left(A_{0}\right)$. Furthermore the spectral problems associated with $A_{\varepsilon}$ converge in the following sense: let $\left(\mu_{i}^{\varepsilon}, v_{i}^{\varepsilon}\right)$ and $\left(\mu_{i}, v_{i}\right)$ be such that

$$
\begin{array}{ccc}
v_{i}^{\varepsilon} \in H_{\varepsilon}, \quad A_{\varepsilon} v_{i}^{\varepsilon}=\mu_{i}^{\varepsilon} v_{i}^{\varepsilon}, \mu_{0}^{\varepsilon} \leq \mu_{1}^{\varepsilon} \leq \ldots \leq \mu_{i}^{\varepsilon}, \ldots, \quad\left(v_{i}^{\varepsilon} \mid v_{j}^{\varepsilon}\right)_{\varepsilon}=\delta_{i, j} \\
v_{i} \in H, \quad A_{0} v_{i}=\mu_{i} v_{i}, \quad \mu_{0} \leq \mu_{1} \leq \ldots \leq \mu_{i}, \ldots, \quad\left(v_{i} \mid v_{j}\right)=\delta_{i, j} .
\end{array}
$$

Then, as $\varepsilon \rightarrow 0, \mu_{i}^{\varepsilon} \rightarrow \mu_{i}$ for every $i \in \mathbb{N}$. Moreover, up to a subsequence, $\left\{v_{i}^{\varepsilon}\right\}$ converges strongly to eigenvectors associated to $\mu_{i}$. Conversely any eigenvector $v_{i}$ is the strong limit of a particular sequence of eigenvectors of $A_{\varepsilon}$ associated to $\mu_{i}^{\varepsilon}$.

Applying this general result to the sequence $\left\{G_{\varepsilon}\right\}$ defined by (3.6), we deduce, in Section 4 , the limit of the shifted spectrum $\left\{\mu_{i}^{\varepsilon}\right\}$, where $\mu_{i}^{\varepsilon}=\lambda_{i}^{\varepsilon}-\frac{\lambda_{0}}{\varepsilon^{2}}$. Let us emphasize that, as already noticed in a similar situation in [1], see Theorem 4.1, the equi-compactness property (ii) is crucial, otherwise, we could only expect the inclusion of the spectrum of $A_{0}$ in the set of cluster points of $\left\{\mu_{i}^{\varepsilon}\right\}$.

Proof. Let $c>c_{0}$. The condition (i) and (3.7) imply that, for small $\varepsilon$, the operator $A_{\varepsilon}+c \mathrm{I}_{H_{\varepsilon}}$, where $\mathrm{I}_{H_{\varepsilon}}$ denotes the identity map on $H_{\varepsilon}$, is a positive maximal monotone. Let us denote by $S_{\varepsilon}$ its inverse. Since $G_{\varepsilon}$ $\Gamma$-converges to $G$, it is easy to check that $G$ is a quadratic lower semicontinuous functional on $H$ which satisfies condition (i) as well. Therefore, for every $f \in H$, the minimum problem:

$$
\inf \left\{G(v)+c\|v\|_{H}^{2}-2(f \mid v): v \in H\right\}
$$

admits a unique minimizer $S_{0} f$ and the map $f \mapsto S_{0} f$ determines a bounded linear operator. The range of $S_{0}$ coincides with the domain $D\left(A_{0}\right)$ of a closed operator $A_{0}: H \mapsto H$ such that $S_{0}=\left(A_{0}+c \mathrm{I}_{H}\right)^{-1}$. 
We claim that $\left\{S_{\varepsilon}\right\}$ is a uniformly compact family of self adjoint operators on $H_{\varepsilon}$ and that $S_{\varepsilon} \rightarrow S_{0}$ strongly. Recalling that, due to (3.7), the spaces $H_{\varepsilon}$ share the same topology, this means that

$$
\sup \left\|f_{\varepsilon}\right\|<+\infty \Rightarrow\left\{S_{\varepsilon} f_{\varepsilon}\right\} \text { strongly relatively compact in } H, S_{\varepsilon} f_{\varepsilon} \rightarrow S_{0} f \text { whenever } f_{\varepsilon} \rightarrow f \text {. }
$$

The conclusions of Theorem 3.1 will then follow from [6], Theorems 11.4 and 11.5 (see also [3]), after noticing that, for $v \in H$, the following equivalences hold:

$$
A_{\varepsilon} v=\mu_{i}^{\varepsilon} v \quad \Longleftrightarrow \quad S_{\varepsilon} v=\frac{1}{\mu_{i}^{\varepsilon}+c} v, \quad A_{0} v=\mu_{i} v \quad \Longleftrightarrow \quad S_{0} v=\frac{1}{\mu_{i}+c} v .
$$

To prove the claim, it is enough to show that for every weakly convergent sequence $f_{\varepsilon}$, the following implication holds

$$
f_{\varepsilon} \rightarrow f \quad \Longrightarrow \quad S_{\varepsilon} f_{\varepsilon} \rightarrow S_{0} f \quad \text { (strongly). }
$$

The crucial remark is that $v_{\varepsilon}$ is the unique minimizing point of $\tilde{G}_{\varepsilon}$ where

$$
\tilde{G}_{\varepsilon}(v):=G_{\varepsilon}(v)+c(v \mid v)_{\varepsilon}-2\left(f_{\varepsilon} \mid v\right)_{\varepsilon} .
$$

Since $\left\{f_{\varepsilon}\right\}$ is bounded, there exists $M>0$ such that

$$
\left(c-c_{0}\right)\left\|v_{\varepsilon}\right\|^{2}-M\left\|v_{\varepsilon}\right\| \leq \tilde{G}_{\varepsilon}\left(v_{\varepsilon}\right) \leq 0 .
$$

It follows that $\left\{v_{\varepsilon}\right\}$ is bounded and that $\sup G_{\varepsilon}\left(v_{\varepsilon}\right)<+\infty$ Therefore, by the condition (ii), $\left\{v_{\varepsilon}\right\}$ is strongly relatively compact.

On the other hand, $\tilde{G}_{\varepsilon}$ being a uniformly convergent perturbation of $G_{\varepsilon}$, it is easy to check that $\tilde{G}_{\varepsilon}$ does $\Gamma-$ converge to the functional $\tilde{G}:=G+k\|\cdot\|^{2}-2(f \mid \cdot)$. Therefore, by using the fundamental variational property of the $\Gamma$-convergence, we derive that $v_{\varepsilon}$ converges to a global minimizer of $\tilde{G}$. This minimizer is unique and coincides with $S_{0} f$. The claim (3.11) follows and Theorem 3.1 is proved.

\section{Convergence Results}

In this section we are going to prove that we can apply Theorem 3.1 to the sequence $\left\{G_{\varepsilon}\right\}$ defined by (3.6) and with $G$ defined as follows

$$
G(v):= \begin{cases}G_{0}(w) & \text { if } v(s, y)=w(s) u_{0}(y), w \in H_{0}^{1}(0, L) \\ +\infty & \text { otherwise }\end{cases}
$$

where

$$
G_{0}(w):=\int_{0}^{L}\left\{\left|w^{\prime}(s)\right|^{2}+\left[\left(\tau(s)+\alpha^{\prime}(s)\right)^{2} C(\omega)-\frac{k^{2}(s)}{4}\right]|w(s)|^{2}\right\} \mathrm{d} s .
$$

Here $u_{0}$ is the normalized eigenvector (ground state) of the unperturbed problem introduced in (3.2) and the geometric parameter $C(\omega)$ is given by

$$
C(\omega):=\int_{\omega}\left|\nabla_{y} u_{0} \cdot R y\right|^{2} \mathrm{~d} y .
$$

Notice that $C(\omega)>0$, unless $u_{0}$ is radial. This parameter which depends only on the shape of the section $\omega$ turns out to be very important as it will govern the effect of the torsion. 


\subsection{A perturbed spectral problem in the cross section}

The influence of the curvature $k(s)$ goes through the multiplicative coefficient $\beta_{\varepsilon}(s, y)$ which appears in (3.6) (that is $\int_{\omega} \beta_{\varepsilon}(s, y)\left(\left|\nabla_{y} v\right|^{2}-\lambda_{0}|v|^{2}\right) \mathrm{d} y$ ). In order to study this dependence, we consider, for every $\xi \in \mathbb{R}^{2}$, the following perturbed problem:

$$
-\operatorname{div}\left[(1-\xi \cdot y) \nabla_{y} u\right]=\lambda(1-\xi \cdot y) u, \quad u \in H_{0}^{1}(\omega)
$$

The parameter $\xi$ will be taken to be $\xi=\varepsilon k(s) z_{\alpha}$ so that for small $\varepsilon$ the perturbed operator is positive with compact resolvent. Let us denote by $\lambda(\xi)>0$ its first eigenvalue, that is:

$$
\lambda(\xi)=\inf _{\substack{v \in H_{0}^{1}(\omega) \\ v \neq \equiv 0}} \frac{\int_{\omega}(1-\xi \cdot y)\left(\nabla_{y} u\right)^{2} \mathrm{~d} y}{\int_{\omega}(1-\xi \cdot y)(u)^{2} \mathrm{~d} y} .
$$

Let $v \in H_{0}^{1}\left(Q_{L}\right)$; then the following lower bound holds for a.e. $s \in(0, L)$ :

$$
\frac{1}{\varepsilon^{2}} \int_{\omega} \beta_{\varepsilon}(s, y)\left(\left|\nabla_{y} v\right|^{2}-\lambda_{0}|v|^{2}\right) \mathrm{d} y \geq \gamma_{\varepsilon}(s) \int_{\omega} \beta_{\varepsilon}(s, y)|v|^{2} \mathrm{~d} y,
$$

where

$$
\gamma_{\varepsilon}(s):=\frac{\lambda\left(\varepsilon k(s) z_{\alpha}(s)\right)-\lambda_{0}}{\varepsilon^{2}}
$$

The fact that $\gamma_{\varepsilon}$ remains finite is crucial in order to find a finite $\Gamma$-limit for $G_{\varepsilon}$. This is also closely related to the validity of the postulated behavior given in (3.3).

Proposition 4.1. (i) The function $\lambda(\xi)$ is twice differentiable at 0 and denoting by I the identity matrix, there holds:

$$
\lambda(0)=\lambda_{0}, \quad \nabla \lambda(0)=0, \quad \nabla^{2} \lambda(0)=-\frac{1}{2} I .
$$

(ii) Let $\gamma_{\varepsilon}(s)$ be given by (4.6) and assume that the curvature $k(s)$ is bounded. Then, as $\varepsilon \rightarrow 0$

$$
\gamma_{\varepsilon}(s) \rightarrow-\frac{k^{2}(s)}{4} \quad \text { uniformly on }[0, L]
$$

Remark 4.2. It is rather suprising that the Hessian matrix found in the assertion (i) is scalar and independent of the shape of the cross section $\omega$. In fact this situation is very specific to the Laplace operator. If we deal with a more general diffusion operator $-\operatorname{div}(a(y) \nabla \cdot)$, being $a(y)$ a non constant positive coefficient, the situation would be quite different. In fact, if in the definition of $\lambda(\xi)$, we replace $(4.4)$ by

$$
-\operatorname{div}\left[(1-\xi \cdot y) a(y) \nabla_{y} u\right]=\lambda(1-\xi \cdot y) u, \quad u \in H_{0}^{1}(\omega)
$$

it turns out that, although the function $\lambda(\cdot)$ is still locally concave in the neighborhood of 0 , the symmetric negative matrix $\nabla^{2} \lambda(0)$ is not, in general, a multiple of the identity matrix. Moreover, and this is the main point, the gradient at 0 does not vanish anymore. This gradient is given by $\nabla \lambda(0)=-2 \int_{\omega} a(y) u_{0} \nabla_{y} u_{0} \mathrm{~d} y$ where now $u_{0}$ is the eigenvector (bound state) of the new unperturbed problem.

In order to prove Proposition 4.1, we introduce, for fixed $\xi \in \mathbb{R}^{2}$, the solution $u_{\xi} \in H_{0}^{1}(\omega)$ of the following problem:

$$
-\Delta u_{\xi}-\lambda_{0} u_{\xi}=-\xi \cdot \nabla_{y} u_{0}, \quad u_{\xi} \perp u_{0} \text { in } L^{2}(\omega) .
$$

The existence of $u_{\xi}$ falls under Fredholm alternative. Since $\lambda_{0}$ is simple, it is enough to observe that the right hand-side in (4.7) is orthogonal to $u_{0}$, which is clear from the fact that $u_{0} \nabla_{y} u_{0}=\frac{1}{2} \nabla_{y} u_{0}^{2}$ has zero mean value 
by the Dirichlet condition on $\partial \omega$. Futhermore, by linearity, denoting by $\chi_{1}, \chi_{2}$ the solutions of (4.7) for $\xi=e_{1}$ and $\xi=e_{2}$, respectively, we have:

$$
u_{\xi}=\xi_{1} \chi_{1}+\xi_{2} \chi_{2} .
$$

Lemma 4.3. For every $\xi \in \mathbb{R}^{2}$, we have

$$
\inf _{v \in H_{0}^{1}(\omega)} \int_{\omega}\left[\left|\nabla_{y} v\right|^{2}-\lambda_{0}|v|^{2}+2\left(\xi \cdot \nabla_{y} u_{0}\right) v\right] \mathrm{d} y=-\frac{|\xi|^{2}}{4} .
$$

Furthermore, the above infimum is reached for $u_{\xi}$ given in (4.7).

Proof. The variational problem in (4.9) is convex and $v$ is a minimizer if and only if it solves the Euler equation: $-\Delta v-\lambda_{0} v=-\xi \cdot \nabla_{y} u_{0}$. Therefore, by (4.7) the minimum is reached at $u_{\xi}$. By the equi-repartition of energy, we are then reduced to check the equality

$$
\int_{\omega}\left(\xi \cdot \nabla_{y} u_{0}\right) u_{\xi}=-\frac{|\xi|^{2}}{4} .
$$

We notice that $u_{0} \nabla_{y} u_{\xi}-u_{\xi} \nabla u_{0}=\nabla_{y}\left(u_{0} u_{\xi}\right)$ has zero mean value and that, by (3.2) and (4.7),

$$
\operatorname{div}\left(u_{0} \nabla_{y} u_{\xi}-u_{\xi} \nabla_{y} u_{0}\right)=u_{0} \Delta u_{\xi}-u_{0} \Delta u_{0}=\left(\xi \cdot \nabla_{y} u_{0}\right) u_{0} .
$$

Then (4.10) follows after integrating twice by parts:

$$
\begin{aligned}
\int_{\omega}\left(\xi \cdot \nabla_{y} u_{0}\right) u_{\xi} \mathrm{d} y & =\frac{1}{2} \int_{\omega}\left[\left(\xi \cdot \nabla_{y} u_{0}\right) u_{\xi}-\left(\xi \cdot \nabla_{y} u_{\xi}\right) u_{0}\right] \mathrm{d} y \\
& =\frac{1}{2} \int_{\omega} \nabla_{y}(\xi \cdot y)\left(u_{\xi} \nabla_{y} u_{0}-u_{0} \nabla_{y} u_{\xi}\right) \mathrm{d} y \\
& =\frac{1}{2} \int_{\omega}(\xi \cdot y) \operatorname{div}\left[u_{0} \nabla_{y} u_{\xi}-u_{\xi} \nabla_{y} u_{0}\right] \mathrm{d} y \\
& =\frac{1}{2} \int_{\omega}(\xi \cdot y)\left(\xi \cdot \nabla_{y} u_{0}\right) u_{0} \mathrm{~d} y \\
& =-\frac{1}{4} \int_{\omega} \operatorname{div}[(\xi \cdot y) \xi] u_{0}^{2} \mathrm{~d} y \\
& =-\frac{1}{4} \int_{\omega}|\xi|^{2} u_{0}^{2} \mathrm{~d} y=-\frac{1}{4}|\xi|^{2} .
\end{aligned}
$$

Proof of Proposition 4.1. In view of definition (4.6) assertions (i) and (ii) will be obtained by proving that:

$$
\lim _{\xi \rightarrow 0} \frac{\lambda(\xi)-\lambda_{0}}{|\xi|^{2}}=-\frac{1}{4}
$$

In order to obtain (4.11) and recalling the definition of $\lambda(\xi)$, we evaluate the Rayleigh quotient $R_{\xi}(u)=\frac{A(u)}{B(u)}$ where

$$
A(u):=\int_{\omega}(1-\xi \cdot y)\left|\nabla_{y} u\right|^{2} \mathrm{~d} y, \quad B(u):=\int_{\omega}(1-\xi \cdot y)|u|^{2} \mathrm{~d} y,
$$

and $u$ is written as $u=t u_{0}+\varphi$ where $t \in \mathbb{R}, \varphi \in H_{0}^{1}(\omega)$ and $\varphi \perp u_{0}$ in $L^{2}(\omega)$. First we notice that, by integrating by parts $\int_{\omega} \nabla_{y} u_{0} \cdot \nabla_{y}((\xi \cdot y) w) \mathrm{d} y$ and taking (3.2) into account, we have for every $w \in H_{0}^{1}(\omega)$ :

$$
\int_{\omega}(\xi \cdot y)\left(\nabla_{y} u_{0} \cdot \nabla_{y} w-\lambda_{0} u_{0} w\right) \mathrm{d} y=-\int_{\omega}\left(\nabla_{y} u_{0} \cdot \xi\right) w \mathrm{~d} y
$$


In particular, using this relation for $w=u_{0}$ and $w=\varphi$, we easily deduce that

$$
A(u)-\lambda_{0} B(u)=\int_{\omega}(1-\xi \cdot y)\left(\left|\nabla_{y} \varphi\right|^{2}-\lambda_{0}|\varphi|^{2}\right) \mathrm{d} y+2 t \int_{\omega}\left(\nabla_{y} u_{0} \cdot \xi\right) \varphi \mathrm{d} y .
$$

Upper bound. We take $u=u_{0}+u_{\xi}$, i.e. $t=1$ and $\varphi=u_{\xi}$, where $u_{\xi}$ is defined by (4.7). In view of (4.8) and (4.12) there is a constant $C>0$, depending only on $\omega$ such that, for $\xi$ small enough,

$$
\left|\int_{\omega}(\xi \cdot y)\left(\left|\nabla_{y} u_{\xi}\right|^{2}-\lambda_{0}\left|u_{\xi}\right|^{2}\right) \mathrm{d} y\right| \leq C|\xi|^{3}, \quad\left|B\left(u_{0}+u_{\xi}\right)-1\right| \leq C|\xi| .
$$

Also by Lemma 4.3, we have $\int_{\omega}\left(\left|\nabla_{y} u_{\xi}\right|^{2}-\lambda_{0}\left|u_{\xi}\right|^{2}\right) \mathrm{d} y+2 \int_{\omega}\left(\nabla_{y} u_{0} \cdot \xi\right) u_{\xi} \mathrm{d} y=-\frac{|\xi|^{2}}{4}$. Consequently, we deduce the following upper bound:

$$
\frac{\lambda(\xi)-\lambda_{0}}{|\xi|^{2}} \leq \frac{A\left(u_{0}+u_{\xi}\right)-\lambda_{0} B\left(u_{0}+u_{\xi}\right)}{|\xi|^{2} B\left(u_{0}+u_{\xi}\right)} \leq \frac{-\frac{1}{4}+C|\xi|}{1-C|\xi|}
$$

Lower bound. We may choose the constant C large enough and $|\xi|$ small enough so that

$$
1-\xi \cdot y \geq 1-C|\xi| \geq 0 \quad \text { in } \omega, \quad B\left(t u_{0}+\varphi\right) \geq(1-C|\xi|) t^{2},
$$

for every $t \in \mathbb{R}, \varphi \in H_{0}^{1}(\omega)$ with $\varphi \perp u_{0}$ in $L^{2}(\omega)$. Now we are going to show that, for a suitable positive real $s_{0}$, the negative part of $\frac{\lambda(\xi)-\lambda_{0}}{|\xi|^{2}}$ satisfies

$$
\left(\frac{\lambda(\xi)-\lambda_{0}}{|\xi|^{2}}\right)^{-} \leq \frac{1}{4(1-C|\xi|)^{2}}+\frac{2 C \lambda_{0} s_{0}^{2}|\xi|}{(1-C|\xi|)}
$$

Indeed, recalling (4.12) and applying (4.9) with $\xi$ substituted by $\frac{t \xi}{(1-C|\xi|)}$, we have

$$
\begin{aligned}
\left(A-\lambda_{0} B\right)\left(t u_{0}+\varphi\right) & \geq(1-C|\xi|)\left[\int_{\omega}\left(\left|\nabla_{y} \varphi\right|^{2}-\lambda_{0}|\varphi|^{2}\right) \mathrm{d} y+\frac{2 t}{(1-C|\xi|)} \int_{\omega}\left(\nabla_{y} u_{0} \cdot \xi\right) \varphi \mathrm{d} y\right] \\
& -2 C \lambda_{0}|\xi| \int_{\omega}|\varphi|^{2} \mathrm{~d} y \\
& \geq \frac{-t^{2}|\xi|^{2}}{4(1-C|\xi|)}-2 C \lambda_{0}|\xi|\|\varphi\|_{L^{2}(\omega)}^{2} .
\end{aligned}
$$

Thus by (4.14):

$$
\frac{\left(R_{\xi}\left(t u_{0}+\varphi\right)-\lambda_{0}\right)^{-}}{|\xi|^{2}} \leq \frac{1}{4(1-C|\xi|)^{2}}+\frac{2 C \lambda_{0} s_{0}^{2}|\xi|}{(1-C|\xi|)}
$$

whenever $s:=\frac{\|\varphi\|_{L^{2}(\omega)}}{|t||\xi|}$ satisfies $s \leq s_{0}$. We claim that, for a suitable choice of $s_{0}$, the left hand member of the previous inequality vanishes for $s \geq s_{0}$. The upper bound (4.15) will follow by taking the supremum with respect to $u=t u_{0}+\varphi$ and then (4.11) is a consequence of (4.13) and (4.15).

We now prove the claim: let $\lambda_{1}$ denote the second eigenvalue of the Laplace operator in the cross section $\omega$; then $\lambda_{1}>\lambda_{0}$ and since $\varphi \perp u_{o}$, we have $\int_{\omega}|\nabla \varphi|^{2} \geq \lambda_{1} \int_{\omega}|\varphi|^{2}$. By (4.17) and Cauchy-Schwartz inequality, it follows that :

$$
\begin{aligned}
\left(A-\lambda_{0} B\right)\left(t u_{0}+\varphi\right) & \geq\left(\lambda_{1}-\lambda_{0}-C\left(\lambda_{1}+\lambda_{0}\right)|\xi|\right)\|\varphi\|_{L^{2}(\omega)}^{2}-2\left\|\nabla_{y} u_{0}\right\|_{L^{2}(\omega)}|t||\xi|\|\varphi\|_{L^{2}(\omega)} \\
& \geq|t|^{2}|\xi|^{2}\left[\left(\lambda_{1}-\lambda_{0}-C\left(\lambda_{1}+\lambda_{0}\right)|\xi|\right) s^{2}-2\left\|\nabla_{y} u_{0}\right\|_{L^{2}(\omega)} s\right]
\end{aligned}
$$

yielding clearly that $R_{\xi}\left(u_{0}+t \varphi\right) \geq \lambda_{0}$ for large values of $s$. The proof of Proposition 4.1 is achieved. 


\subsection{The main result}

Recalling the definitions of $G_{\varepsilon}$ and $G$ (see (3.6) and (4.1)-(4.3)), we are now in position to state our main result. In what follows we will assume that $k(s), \tau(s)$ belong to $L^{\infty}(0, L)$ and that the angular parameter $\alpha(s)$ is a Lipschitz function. We introduce the effective potential $q(s) \in L^{\infty}(0, L)$ given by

$$
q(s):=\left(\tau(s)+\alpha^{\prime}(s)\right)^{2} C(\omega)-\frac{k(s)^{2}}{4} .
$$

\section{Theorem 4.4.}

(i) The sequence $\left\{G_{\varepsilon}\right\}$ satisfies all conditions (i), (ii), (iii) of Theorem 3.1 .

(ii) The eigenvalues $\lambda_{i}^{\varepsilon}$ of the spectral problem (3.1) satisfy (3.3) where $\mu_{i}(i \in \mathbb{N})$ are the eigenvalues of the following Sturm-Liouville problem

$$
-\varphi^{\prime \prime}+q(s) \varphi=\mu \varphi, \quad \varphi \in H_{0}^{1}(0, L),
$$

(iii) Let $u_{i}^{\varepsilon}$ be a normalized eigenvector for problem (3.1) associated with $\lambda_{i}^{\varepsilon}\left(\right.$ recall $\left.\left(u_{i}^{\varepsilon} \mid u_{j}^{\varepsilon}\right)=\delta_{i, j}\right)$. Then, possibly after extraction of a subsequence, $v_{i}^{\varepsilon}=\psi_{\varepsilon}\left(u_{i}^{\varepsilon}\right)$ converges strongly in $L^{2}\left(Q_{L}\right)$ to $v_{i}(s, y)=$ $w_{i}(s) u_{0}(y)$ where $w_{i}$ is a normalized eigenvector of problem $(4.17),(4.18)$ associated with $\mu_{i}$. Conversely, any such $v_{i}$ is the limit of a sequence $\psi_{\varepsilon}\left(u_{\varepsilon}\right)$ where $u_{\varepsilon}$ is an eigenvector of (3.1) associated with $\lambda_{i}^{\varepsilon}$.

Remark 4.5. In the particular case of a circular cross section of radius $R$, the ground state $u_{0}$ is radial: it is given by $u_{0}(x)=\frac{\sqrt{2}}{R J_{1}\left(\sqrt{\lambda_{0}} R\right)} J_{0}\left(\sqrt{\lambda_{0}}|x|\right)$ where $\lambda_{0}=\left(\frac{r_{0}}{R}\right)^{2},|x|$ is the distance to the axis, $J_{0}, J_{1}$ are the first and second Bessel functions and $r_{0}$ denote the first zero of $J_{0}$. Therefore the constant $C(\omega)$ defined in (4.3) vanishes and we recover, by variational methods, the curvature dependence obtained in [5] (in [2,5], the length $L$ is infinite and the method used is based on formal operator asymptotic expansions).

In this paper we bring to the fore a new effect due to torsion. This effect appears when the constant $C(\omega)$ is stricly positive; this is the case for example if $\omega$ is a rectangle. Then the rotation of the section with respect to the Tang frame $\left(\tau+\alpha^{\prime}\right)$ produces a shift of the spectrum on the right. This effect comes into competition with the curvature effect which produces a shift on the left.

Remark 4.6. The tools used in our approach are very flexible and we may as well deal with Neumann conditions on the extremities of the tube (which after the change of variables corresponds to $\{0, L\} \times \omega)$ and the limit spectral equation (4.17) would be changed accordingly.

Remark 4.7. The case of a heterogeneous medium decribed by a diffusion coefficient $a(y)$ (see Rem. 4.2), for example $a(y)$ taking two values and jumping across a coaxial cylinder, is beyond the scope of this paper. In that case the behavior of the eignevalues as $\varepsilon \rightarrow 0$ include in general an additional term of order $\varepsilon^{-1}$ and the asymptotic behavior given in (3.3) has to be replaced by

$$
\lambda_{i}^{\varepsilon}=\frac{\lambda_{0}}{\varepsilon^{2}}+\frac{\lambda_{1}}{\varepsilon}+\mu_{i}+\rho(\varepsilon), \quad \lim _{\varepsilon \rightarrow 0} \rho(\varepsilon)=0
$$

The presence of the non zero coeffient $\lambda_{1}$ is a direct consequence of the fact that the gradient of the function $\lambda(\xi)$, introduced in Section 4.1, does not vanish, as emphasized in Remark 4.2.

Remark 4.8. In order to modelize a tube with infinite length, it is natural to send $L \rightarrow+\infty$ (after substituting the interval $(0, L)$ by $(-L / 2, L / 2))$. Doing so, we expect from $(4.17),(4.18)$ that the corresponding spectral set $\left\{\mu_{i, L}\right\}$ will converge to the spectrum of the same operator on the whole real line i.e. $-w^{\prime \prime}+q(s) w, w \in L^{2}(\mathbb{R})$. Now it is possible to proceed in different ways namely as in [5] by considering for every $\varepsilon$ the spectral problem on the infinite tube and then passing to the limit in $\varepsilon$. It seems to us that both ways lead to the same limit operator. The proof of this fact would require futher analysis and, in particular, a generalization of our results in which we could choose the length $L=L(\varepsilon)$ to be dependant of $\varepsilon$ with $L_{\varepsilon} \rightarrow \infty$. 


\subsection{Proof of the main theorem}

We proceed in four steps: in Step 1, we prove that $\left\{G_{\varepsilon}\right\}$ satisfies the conditions (i), (ii) of Theorem 3.1. Then to prove the $\Gamma$-convergence result (condition (iii)), we establish the lower bound inequality (Step 2) and the upper bound inequality (Step 3). In the last step, we apply Theorem 3.1.

Step 1. Recalling the definition of $G_{\varepsilon}$ in (3.6), we deduce from (4.5) the lower bound

$$
G_{\varepsilon}(v) \geq \int_{0}^{L} \int_{\omega}\left\{\frac{1}{\beta_{\varepsilon}}\left|v^{\prime}+\nabla_{y} v \cdot R y\left(\tau+\alpha^{\prime}\right)\right|^{2}+\beta_{\varepsilon}(y, s) \gamma_{\varepsilon}(s)|v|^{2}\right\} \mathrm{d} y \mathrm{~d} s .
$$

Accordingly, the inequality i) is satisfied for any $c_{0}$ so large that $\operatorname{essinf}_{Q_{L}}\left\{\gamma_{\varepsilon} \beta_{\varepsilon}\right\} \geq-c_{0}$. Since the curvature $k(s)$ belongs to $L^{\infty}(0, L), \beta_{\varepsilon} \rightarrow 1$ uniformly on $Q_{L}$ (see (3.5)) whereas by Proposition $4.1 \gamma_{\varepsilon}(s) \rightarrow-\frac{1}{4} k^{2}(s)$ uniformly. The latter lower bound is achieved, for $\varepsilon$ small enough, provided $c_{0}>\frac{1}{4}\left(\|k\|_{\infty}\right)^{2}$.

Consider now a sequence $\left\{v_{\varepsilon}\right\}$ bounded in $L^{2}\left(Q_{L}\right)$ such that $G_{\varepsilon}\left(v_{\varepsilon}\right) \leq M$. Then, as $\beta_{\varepsilon}$ is unifomly close to 1 , we infer from (4.19) that:

$$
\limsup _{\varepsilon \rightarrow 0} \int_{Q_{L}}\left|v_{\varepsilon}^{\prime}+\nabla_{y} v_{\varepsilon} \cdot R y\left(\tau+\alpha^{\prime}\right)\right|^{2} \leq C<+\infty
$$

and from (3.6) that

$$
\begin{aligned}
\limsup _{\varepsilon \rightarrow 0} \int_{Q_{L}}\left|\nabla_{y} v_{\varepsilon}\right|^{2} & \leq \limsup _{\varepsilon \rightarrow 0} \int_{Q_{L}} \beta_{\varepsilon}\left|\nabla_{y} v_{\varepsilon}\right|^{2} \\
& \leq \limsup _{\varepsilon \rightarrow 0}\left\{\int_{Q_{L}} \beta_{\varepsilon}\left(\left|\nabla_{y} v_{\varepsilon}\right|^{2}-\lambda_{0}\left|v_{\varepsilon}\right|^{2}\right)+\lambda_{0} \int_{Q_{L}} \beta_{\varepsilon}\left|v_{\varepsilon}\right|^{2}\right\} \\
& \leq \limsup _{\varepsilon \rightarrow 0}\left\{C \varepsilon^{2}+\lambda_{0} \limsup _{\varepsilon \rightarrow 0} \int_{Q_{L}}\left|v_{\varepsilon}\right|^{2}\right\}<+\infty
\end{aligned}
$$

From (4.20), (4.21) and the fact that $\tau+\alpha^{\prime}$ is bounded, we infer that the sequence $\left\{D v_{\varepsilon}\right\}$, where $D v_{\varepsilon}=$ $\left(v_{\varepsilon}^{\prime}, \nabla_{y} v_{\varepsilon}\right)$, is bounded in $L^{2}\left(Q_{L}\right)$. Thus $\left\{v_{\varepsilon}\right\}$ is bounded in $H_{0}^{1}\left(Q_{L}\right)$ and strongly relatively compact in $L^{2}\left(Q_{L}\right)$ by Rellich-Kondrachov Theorem.

Step 2. Let $\left\{v_{\varepsilon}\right\}$ be a sequence such that $v_{\varepsilon} \rightarrow v$ in $L^{2}\left(Q_{L}\right)$. Up to a subsequence we may assume that $\liminf _{\varepsilon \rightarrow 0} G_{\varepsilon}\left(v_{\varepsilon}\right)=\lim _{\varepsilon \rightarrow 0} G_{\varepsilon}\left(v_{\varepsilon}\right)<+\infty$. Then, as proved in Step 1, the sequence is bounded in $H_{0}^{1}\left(Q_{L}\right)$ and inequalities (4.20) and (4.21) apply. Therefore, $v$ belongs to $H_{0}^{1}\left(Q_{L}\right)$ and $v_{\varepsilon}^{\prime} \rightarrow v^{\prime}, \nabla_{y} v_{\varepsilon} \rightarrow \nabla_{y} v$ weakly in $L^{2}\left(Q_{L}\right)$. In particular, as $R y\left(\tau+\alpha^{\prime}\right) \in L^{\infty}\left(Q_{L}\right)$, we obtain:

$$
v_{\varepsilon}^{\prime}+\nabla_{y} v_{\varepsilon} \cdot R y\left(\tau+\alpha^{\prime}\right) \rightarrow v^{\prime}+\nabla_{y} v \cdot R y\left(\tau+\alpha^{\prime}\right)
$$

Futhermore, from (4.19) and the uniform convergence of $\beta_{\varepsilon} \gamma_{\varepsilon}$ to $-\frac{1}{4} k^{2}(s)$, we deduce that

$$
\liminf _{\varepsilon \rightarrow 0} G_{\varepsilon}\left(v_{\varepsilon}\right) \geq \int_{Q_{L}}\left\{\left|v^{\prime}+\nabla_{y} v \cdot R y\left(\tau+\alpha^{\prime}\right)\right|^{2}-\frac{k^{2}}{4}|v|^{2}\right\} \mathrm{d} y \mathrm{~d} s
$$

Now, due to (4.21) and the strong convergence of $v_{\varepsilon}$, we derive that

$$
\int_{Q_{L}}\left|\nabla_{y} v\right|^{2} \leq \liminf _{\varepsilon \rightarrow 0} \int_{Q_{L}}\left|\nabla_{y} v\right|^{2} \leq \lambda_{0} \limsup _{\varepsilon \rightarrow 0} \int_{Q_{L}}\left|v_{\varepsilon}\right|^{2}=\lambda_{0} \int_{Q_{L}}|v|^{2} .
$$


In other words, we have $\int_{0}^{L} f(s) \mathrm{d} s=0$ where the function $f(s):=\int_{\omega}\left(\left.\nabla_{y} v\right|^{2}-\lambda_{0}|v|^{2}\right)(s, y) \mathrm{d} y$ is nonnegative by the definition of $\lambda_{0}$. Therefore, for a.e. $s \in(0, L), f(s)$ vanishes and $v(s, \cdot)$, as an eigenvector associated with $\lambda_{0}$, is proportional to the ground state $u_{0}$. We deduce that $v$ can be written in the form $v(s, y)=w(s) u_{0}(y)$ with $w \in H_{0}^{1}(0, L)$ (since $v \in H_{0}^{1}\left(Q_{L}\right)$ ). We plug this expression of $v$ into (4.22) and, after straightforward computations where we use (4.3) and the equalities $\int_{\omega} u_{0}^{2} \mathrm{~d} y=1, \int_{\omega} u_{0} \nabla_{y} u_{0} \cdot R y \mathrm{~d} y=0$, we conclude that $\liminf _{\varepsilon \rightarrow 0} G_{\varepsilon}\left(v_{\varepsilon}\right) \geq G(v)$ where $G(v)=G_{0}(w)$ is given by (4.1) (4.2). This concludes the proof of the lower bound part of the $\Gamma$-convergence.

Step 3. Let $v \in L^{2}\left(Q_{L}\right)$. We have to show the existence of a sequence $\left\{v_{\varepsilon}\right\}$ such that $v_{\varepsilon} \rightarrow v$ and $\limsup _{\varepsilon \rightarrow 0} G_{\varepsilon}\left(v_{\varepsilon}\right) \leq G(v)$. We may assume that $G(v)<+\infty$ so that, in view of (4.1), we can write $v(s, y)=$ $w(s) u_{0}(y)$ for a suitable element $w \in H_{0}^{1}(0, L)$. We consider $v_{\varepsilon}$ defined by $v_{\varepsilon}=w(s)\left[u_{0}(y)+\varepsilon \varphi(s, y)\right]$ where $\varphi \in H_{0}^{1}\left(Q_{L}\right)$ will be chosen later. Clearly $v_{\varepsilon} \rightarrow v$ strongly in $H_{0}^{1}\left(Q_{L}\right)$ and, as $\beta_{\varepsilon}$ is uniformly close to 1 , we have

$$
\begin{aligned}
\lim _{\varepsilon \rightarrow 0} \int_{Q_{L}} \frac{1}{\beta_{\varepsilon}}\left|v_{\varepsilon}^{\prime}+\nabla_{y} v_{\varepsilon} \cdot R y\left(\tau+\alpha^{\prime}\right)\right|^{2} \mathrm{~d} s \mathrm{~d} y & =\int_{Q_{L}}\left|v^{\prime}+\nabla_{y} v \cdot R y\left(\tau+\alpha^{\prime}\right)\right|^{2} \mathrm{~d} s \mathrm{~d} y \\
& =\int_{0}^{L}\left|w^{\prime}\right|^{2}+\left[\left(\tau+\alpha^{\prime}\right)(s)^{2} C(\omega)\right]|w|^{2} \mathrm{~d} s .
\end{aligned}
$$

As in the proof of Lemma 4.3, we find that, for a.e. s:

$$
\int_{\omega} \frac{\beta_{\varepsilon}(s, y)}{\varepsilon^{2}}\left(\left|\nabla_{y} v_{\varepsilon}\right|^{2}-\lambda_{0}\left|v_{\varepsilon}\right|^{2}\right) \mathrm{d} y=\int_{\omega} \beta_{\varepsilon}(s, y)\left[\left(\left|\nabla_{y} \varphi\right|^{2}-\lambda_{0}|\varphi|^{2}\right)+2 k(s) z_{\alpha}(s) \cdot \nabla_{y} u_{0} \varphi\right] w^{2}(s) \mathrm{d} y .
$$

Integrating with repect to $s$, passing to the limit as $\varepsilon \rightarrow 0$ and taking into account (4.23), we are led to

$$
\lim _{\varepsilon \rightarrow 0} G_{\varepsilon}\left(v_{\varepsilon}\right)=\int_{0}^{L}\left|w^{\prime}\right|^{2}+\left[\left(\tau+\alpha^{\prime}\right)(s)^{2} C(\omega)\right]|w|^{2} \mathrm{~d} s+F(\varphi)
$$

where

$$
F(\varphi):=\int_{Q_{L}}\left[\left(\left|\nabla_{y} \varphi\right|^{2}-\lambda_{0}|\varphi|^{2}\right)+2 k(s) z_{\alpha}(s) \cdot \nabla_{y} u_{0} \varphi\right] w^{2}(s) \mathrm{d} s \mathrm{~d} y .
$$

We observe that $F$, as a functional on $H_{0}^{1}\left(Q_{L}\right)$, is derivative free with respect to $s$ and then can be extended by continuity to the larger space $L^{2}\left(0, L ; H_{0}^{1}(\omega)\right)$. Recalling (4.7) and (4.8), and in view of Lemma 4.3, the minimizer of this extended functional $\tilde{F}$ is reached for $\varphi=\varphi_{0}$ where

$$
\varphi_{0}(s, y):=u_{\left(k z_{\alpha}\right)(s)}(y)=k(s)\left[\cos \alpha(s) \chi_{1}(y)-\sin \alpha(s) \chi_{2}(y)\right] .
$$

Therefore

$$
\inf \left\{F(\varphi): \varphi \in H_{0}^{1}\left(Q_{L}\right)\right\}=\tilde{F}\left(\varphi_{0}\right)=-\int_{Q_{L}} \frac{k^{2}(s)}{4} w^{2}(s) \mathrm{d} s .
$$

Now we choose a minimizing sequence $\left\{\varphi_{n}\right\}$ in $H_{0}^{1}\left(Q_{L}\right)$ such that $\varphi_{n} \rightarrow \varphi_{0}$ in $L^{2}\left(0, L ; H_{0}^{1}(\omega)\right)$. Then replacing $v_{\varepsilon}$ by $v_{\varepsilon, n}=w(s)\left(u_{0}(y)+\varepsilon \varphi_{n}(s, y)\right)$ in $(4.24)$, we obtain:

$$
\limsup _{n \rightarrow \infty}\left(\limsup _{\varepsilon \rightarrow 0} G_{\varepsilon}\left(v_{\varepsilon, n}\right)\right) \leq G_{0}(w)(=G(v)) .
$$

The conclusion follows by taking a diagonal subsequence.

Step 4. Assume further that $G \geq-c_{0}\|\cdot\|^{2}$ for a suitable $c_{0} \geq 0$ then, as a functional from $L^{2}\left(Q_{L}\right)$ into $(-\infty,+\infty], \mathrm{G}$ is lower semicontinuous and quadratic (see [5], Th. 11.10). Denote by $V$ the subspace where it is finite. By [4], Theorem 12.13, there exists a bilinear symmetric form $a_{0}(u, v)$ such that $G(v)=a_{0}(v, v)$ if 
$v \in V$ and such that the associated operator $A_{0}: L^{2}\left(Q_{L}\right) \rightarrow L^{2}\left(Q_{L}\right)$ is self adjoint with dense domain (in fact $A_{0}+c_{0} \mathrm{I}_{\mathrm{Q}_{\mathrm{L}}}$ is maximal monotone).

We apply Theorem 3.1 to the sequence $\left\{G_{\varepsilon}\right\}$ which by the previous steps satisfy all the required conditions. The domain of $G$ can be identified with the space $H_{0}^{1}(0, L)$ through the map $v(s, y)=w(s) u_{0}(y) \mapsto w(s)$. In this identification, the self-adjoint operator $A_{0}$ associated with $G$ becomes

$$
A_{0}: w \in H_{0}^{1}(0, L) \cap H^{2}(0, L) \rightarrow-w^{\prime \prime}+q(s) w \in L_{2}(0, L) .
$$

\section{Physical interpretation AND EXAMPles}

As stated in Theorem 4.4, the result obtained can be put in the form of a Sturm-Liouville problem. Equations (4.17) and (4.18) can be interpreted as a onedimensional problem for the spatial wave equation of a particle confined to move in a onedimensional waveguide with a potential given by $q(s)$. In other words, although we have started from a threedimensional problem without a potential in the interior of the domain under consideration, in the limit, in a onedimensional curved waveguide, the particle sees the curvature, the torsion and the influence of the cross section as a (nonhomogeneous) potential function in an equivalent straight waveguide of the same total length. This potential, induced by the geometry of the waveguide, includes the influence of the curvature and of the rotation of the section through the functions $k(s), \alpha(s)$ and $\tau(s)$; also the influence of the shape of the cross section goes through the constant $C(\omega)$, for it depends both on $\omega$ and on the eigenfunction $u_{0}$ which changes with $\omega$. Moreover, as pointed out in Remark 4.5, the effects of curvature and rotation compete against each other.

Assume that, from the start, we have a straight waveguide, that is $k \equiv 0$ and $\tau \equiv 0$. If the cross section is circular (or if $\alpha^{\prime} \equiv 0$ ) then one obtains the classical (eigenvalue, eigenvector) pairs $\left(\left(\frac{n \pi}{L}\right)^{2}, \sqrt{\frac{2}{L}} \sin \frac{n \pi x}{L}\right.$ ).

Assume now that the cross section is a square. Then $C(\omega)>0$ and we can very easily simulate an arbitrary positive potential by suitably choosing the rotation angle $\alpha(s)$. In particular, if we consider a straight waveguide of infinite length twisted so that $\alpha$ is periodic, then our analysis (see Rem. 4.8) allows us to predict the possible presence of band gaps in the limit as $L \rightarrow \infty$ since, by (4.17), we get an effective potential $q(s)=C(\omega)\left(\alpha^{\prime}(s)\right)^{2}$ on the real line which is periodic.

Coming back to a wave guide of finite length $L$, let us now illustrate the change of the probability density function through a simple example. Let us consider that $q$ is constant in a certain interval $[a, L] \subset[0, L]$ and zero in $[0, a[$. Then, in this case, solving (4.16), (4.17) leads to search for $\mu \geq q$ such that:

$$
\sin (\sqrt{\mu} a) \cos [\sqrt{\mu-q}(L-a)]+\frac{\sqrt{\mu}}{\sqrt{\mu-q}} \cos (\sqrt{\mu} a) \sin [\sqrt{\mu-q}(L-a)]=0,
$$

(where by convention $\sqrt{\mu}=i \sqrt{-\mu}$ if $\mu<0$ ).

In Figure 3, we show the dependence of the eigenvalues with respect to $a / L$ for $q=-6$ and $L=2$.

We remark that for $a / L=1$ (or $a / L=0$ ), we must obtain the usual eigenvalues

$$
\mu_{n}=\left(\frac{n \pi}{L}\right)^{2} \quad \text { or } \quad \mu_{n}=\left(\frac{n \pi}{L}\right)^{2}+q \quad(n \in \mathbb{N}) .
$$

If, for example, one chooses $q=-6, a=1$ and $L=2$ one gets $\mu \approx-1.363855334$ and the probability density function $P(s)=w^{*}(s) w(s)$ becomes (Fig. 4).

As is clear from the above example, a local perturbation of the curvature and/or the torsion and/or the shape of the cross section will change not only the energy levels but also the wave function and, consequentely, the probability density function in the waveguide. For example, if one whishes that the probability density function be concentrate near the end $s=L$, then one should strongly bend the waveguide near the end. For the case where one has $q=-80, a=1.8$ and $L=2$ the result is the following (Fig. 5).

Now, owing to $(4.17),(4.18)$ if the shape contant $C(\omega)$ is stricly positive, we may start from the situation depicted in Figure 5 and apply a rotation $\alpha(s)$ of increasing amplitude between $L / 2$ and $L$ in order to compensate 


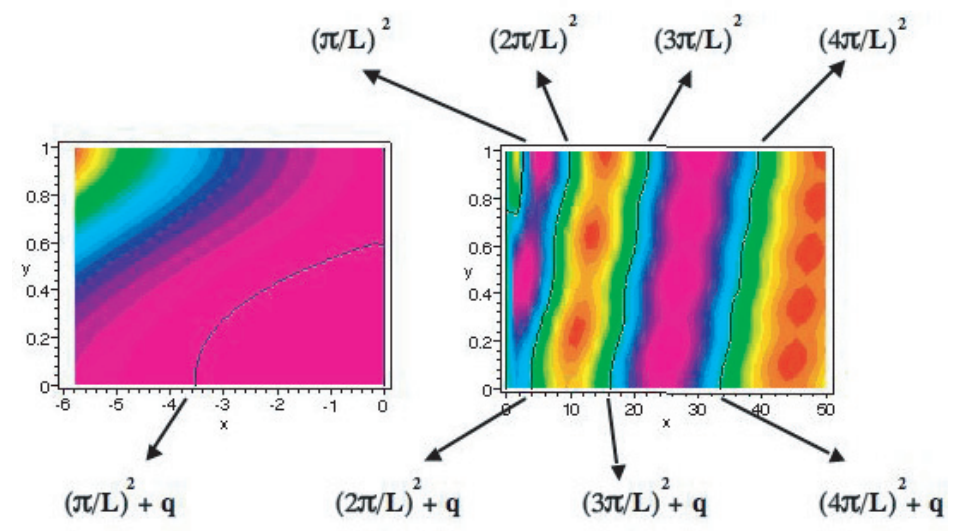

Figure 3. $\mu_{1}, \mu_{2}, \mu_{3}, \mu_{4}$ vs. $a / L$ for $q=-6$ and $L=2$.

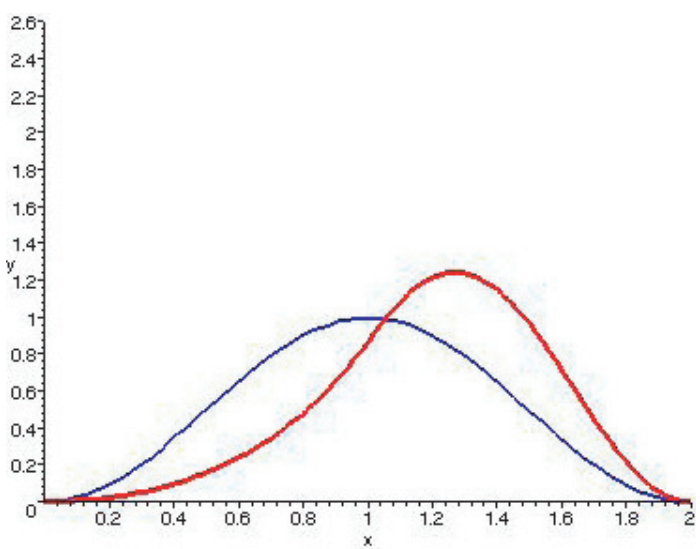

Figure 4. Probability density function (thick line) and for the classical case (thin line) $(q=$ $-6, a=1$ and $L=2$ ).

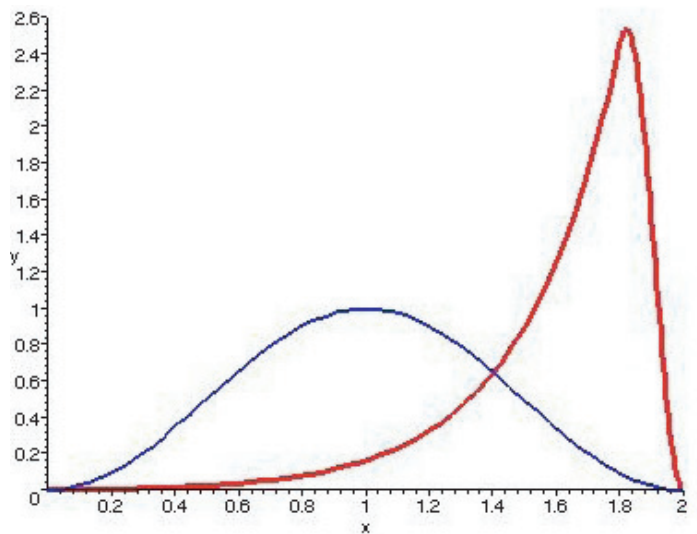

Figure 5. Probability density function (thick line) and for the classical case (thin line) $(q=$ $-80, a=1.8$ and $L=2$ ). 
the curvature and in such a way that we recover the probability density depicted in Figure 4 . In other words, by playing with the curvature and the torsion of the waveguide, we can control the energy levels and bound state solutions at will.

Acknowledgements. This research was partially supported by Program PAI-PESSOA. The research of M.L. Mascarenhas and L. Trabucho was also supported by POCI/Mat/60587/2004. The authors acknowledge the hospitality of the Universities of Sud-Toulon-Var (ANAM) and Lisbon (CMAF), where this work was undertaken. The authors thank P. Seppecher for some enlightening discussion.

\section{REFERENCES}

[1] G. Allaire and C. Conca, Bloch wave homogenization and spectral asymptotic analysis. J. Math. Pures Appl. 77 (1998) 153-208.

[2] B. Chenaud, P. Duclos, P. Freitas and D. Krejčiřík, Geometrically induced discrete specrtum in curved tubes. Differ. Geometry Appl. 23 (2005) 95-105.

[3] C. Conca, J. Planchard and M. Vanninathan, Fluids and periodic structures, Research in Applied Mathematics 38. Masson, Paris (1995).

[4] G. Dal Maso, An Introduction to $\Gamma$-Convergence. Birkhäuser, Boston (1993).

[5] P. Duclos and P. Exner, Curvature-induced bounds states in quantum waveguides in two and tree dimensions. Rev. Math. Phys. 7 (1995) 73-102.

[6] V. Jikov, S.M. Kozlov and O.A. Oleinik, Homogenization of Differential Operators and Integral Equations. Springer-Verlag, Berlin (1994)

[7] P. Kuchment, On some spectral problems of mathematical physics. Partial differential equations and inverse problems., Contemp. Math. 362. Amer. Math. Soc., Providence, RI (2004) 241-276.

[8] J. Rubinstein, M. Schatzman, Variational problems on multiply connected thin strips. II. Convergence of the Ginzburg-Landau functional. Arch. Ration. Mech. Anal. 160 (2001) 309-324.

[9] M. Vanninathan, Homogenization of eigenvalue problems in perforated domains. Proc. Indian Acad. Sci. Math. Sci. 90 (1981) $239-271$ 